Article

\title{
Hot Spots and Their Contribution to the Self-Assembly of the Viral Capsid: In Silico Prediction and Analysis
}

\author{
Armando Díaz-Valle ${ }^{1}$, José Marcos Falcón-González ${ }^{2}$ and Mauricio Carrillo-Tripp ${ }^{1, *(D)}$ \\ 1 Biomolecular Diversity Laboratory, Centro de Investigación y de Estudios Avanzados del Instituto \\ Politécnico Nacional Unidad Monterrey, Vía del Conocimiento 201, Parque PIIT, C.P. 66600 Apodaca, \\ Nuevo León, Mexico; diaz.armando21@gmail.com \\ 2 Unidad Profesional Interdisciplinaria de Ingeniería Campus Guanajuato, Instituto Politécnico Nacional, \\ Av. Mineral de Valenciana No. 200, Col. Fraccionamiento Industrial Puerto Interior, \\ C.P. 36275 Silao de la Victoria, Guanajuato, Mexico; profymmarcos@gmail.com \\ * Correspondence: mauricio.carrillo@cinvestav.mx
}

Received: 12 October 2019; Accepted: 15 November 2019; Published: 27 November 2019

check for updates

\begin{abstract}
The viral capsid is a macromolecular complex formed by a defined number of self-assembled proteins, which, in many cases, are biopolymers with an identical amino acid sequence. Specific protein-protein interactions (PPI) drive the capsid self-assembly process, leading to several distinct protein interfaces. Following the PPI hot spot hypothesis, we present a conservation-based methodology to identify those interface residues hypothesized to be crucial elements on the self-assembly and thermodynamic stability of the capsid. We validate the predictions through a rigorous physical framework which integrates molecular dynamics simulations and free energy calculations by Umbrella sampling and the potential of mean force using an all-atom molecular representation of the capsid proteins of an icosahedral virus in an explicit solvent. Our results show that a single mutation in any of the structure-conserved hot spots significantly perturbs the quaternary protein-protein interaction, decreasing the absolute value of the binding free energy, without altering the protein's secondary nor tertiary structure. Our conservation-based hot spot prediction methodology can lead to strategies to rationally modulate the capsid's thermodynamic properties.
\end{abstract}

Keywords: free energy; structural conservation; functional dimer; protein-protein interaction; site-directed mutagenesis; binding free energy; molecular dynamics; alanine-scanning

\section{Introduction}

The viral capsid is the archetypal molecular system of protein self-assembly and an excellent model for studying protein-protein interaction mechanisms that form symmetric closed shells [1]. The capsid is a macromolecular complex built by capsid proteins (CPs), which are biopolymers that in many cases have an identical amino acid sequence. Capsid formation occurs rapidly and spontaneously with a high degree of fidelity. The molecular mechanism followed by the CPs to form the capsid is not fully understood yet, due to the intrinsic complexity of the required protein-protein interfaces. There is evidence suggesting that the self-assembly process follows encoded signals in the sequence and structure of the CPs to guide the formation of the final virus particle. If correct, such molecular recognition signals are crucial elements for the initial nucleation and subsequent growth of the capsid. Therefore, accurate identification of those molecular signals, the so-called interface hot spots, will shed light on our understanding of the viral self-assembly process, or even macromolecular assembly in general. 


\subsection{Capsid Quaternary Structure}

Viral capsids present either of two quaternary structures with basic symmetries: icosahedral (spherical or isotropic) or helical (rod-shaped, filamentous, or anisotropic). Icosahedral capsids are adopted by more than half of the virus families we currently know. In contrast, helical capsids are found in $\sim 10 \%$ of virus families [2]. Watson and Crick proposed the basic principles for the construction of icosahedral viruses [3]. Then, Caspar and Klug developed the quasi-equivalence theory [4], which has been the foundation of modern structural virology.

In general, the residues of a protein in a monomeric state can be grouped according to their location in the native fold, namely, core residues or surface residues. In principle, the former are responsible for the tertiary structure, and the later are highly solvent-exposed. When a stable quaternary interaction takes place to form a protein complex, a third region is formed, i.e., the interface region. All residues in close contact between the interacting proteins are the interface residues of the complex.

In particular, capsids are formed by a definite number of chemically identical proteins (CPs), also known as subunits. The CPs self-assemble spontaneously, yielding monodispersed particles (Figure 1A). In the case of spherical capsids, the arrangement of the CPs can be inscribed in an icosahedral lattice with symmetrical characteristics, as explained by the Caspar and Klug theory. In such a quaternary structure, each $\mathrm{CP}$ interacts and forms interfaces with all of its neighboring subunits. This geometrical arrangement creates a complex network of interface residues. To simplify the study of such network, its size can be reduced by taking advantage of the particle's symmetries and focusing on just $1 / 60$ th of the capsid, commonly referred to as the icosahedral asymmetric unit (Figure 1B).

\subsection{Protein-Protein Interface Hot Spot Prediction}

Several works have proposed different strategies to predict the location of hot spots in a protein complex. Many of the available methods take advantage of the fact that, by definition, the contribution of each interface residue to the binding free energy is not homogeneous, i.e., some contribute significantly more than others. Commonly, an averaged energy-based alanine scanning mutagenesis approximation method is implemented for this purpose. Even though those methods can make fast calculations, one has to keep in mind that such approximations and physical assumptions will not necessarily produce a reliable result. Examples of this type of predictors are ROBBETA [5], FoldX [6], SpotOn [7], and iPPHOT [8]. Other approaches have opted for machine-learning-based methods [9-12], molecular-dynamics-based methods [13,14], or combining solvent accessibility and inter-residue potentials [15]. Despite these efforts, there is still not a clear way nor strict rule to locate hot spots on a protein complex.

On a previous work, we reported a methodology to map the 3D spatial location of the interface residues involved in all quaternary interactions in a capsid into a 2D representation, or CapsidMap [16,17]. The motivation to build a CapsidMap of a virus was to quantitatively evaluate the quaternary structure similarity between two capsids through a metric (S-score). When comparing capsids of different viruses, we noted the existence of a set of interface residues for the icosahedral Nodaviridae virus family who were conserved not only in sequence but also in quaternary structure among all related members. Furthermore, those structure-conserved interface residues were found to form non-random patterns around the capsid's symmetry axes. Therefore, we propose that other virus families could also present residues with structural conservation characteristics. As a conclusion, in that work, we hypothesized that those residues should be interface hot spots and might have a crucial role in the self-assembly mechanisms due to their evolutionary persistence.

This work has two goals. First, we formally present a conservation-based methodology to locate hot spots. The following three steps define the general pipeline for a given virus family or genus. (i) For each member, identify all the interface residues between capsid subunits; this step is highly simplified by the use of the asymmetric unit and the automatized tools on the VIPERdb Science Gateway [18]. Then, for the whole set, (ii) identify residues conserved in sequence by multiple sequence alignment 
(MSA). Finally, (iii) identify residues conserved in quaternary structure by multiple CapsidMaps alignment. The intersection between the three sets of residues are the predicted conservation-based hot spots of that family or genus (Figure 1C). This is an alternative to other methods, i.e., no energy calculations nor physical approximations are involved in the predictions. Here, we applied such methodology to the Bromoviridae icosahedral virus family.
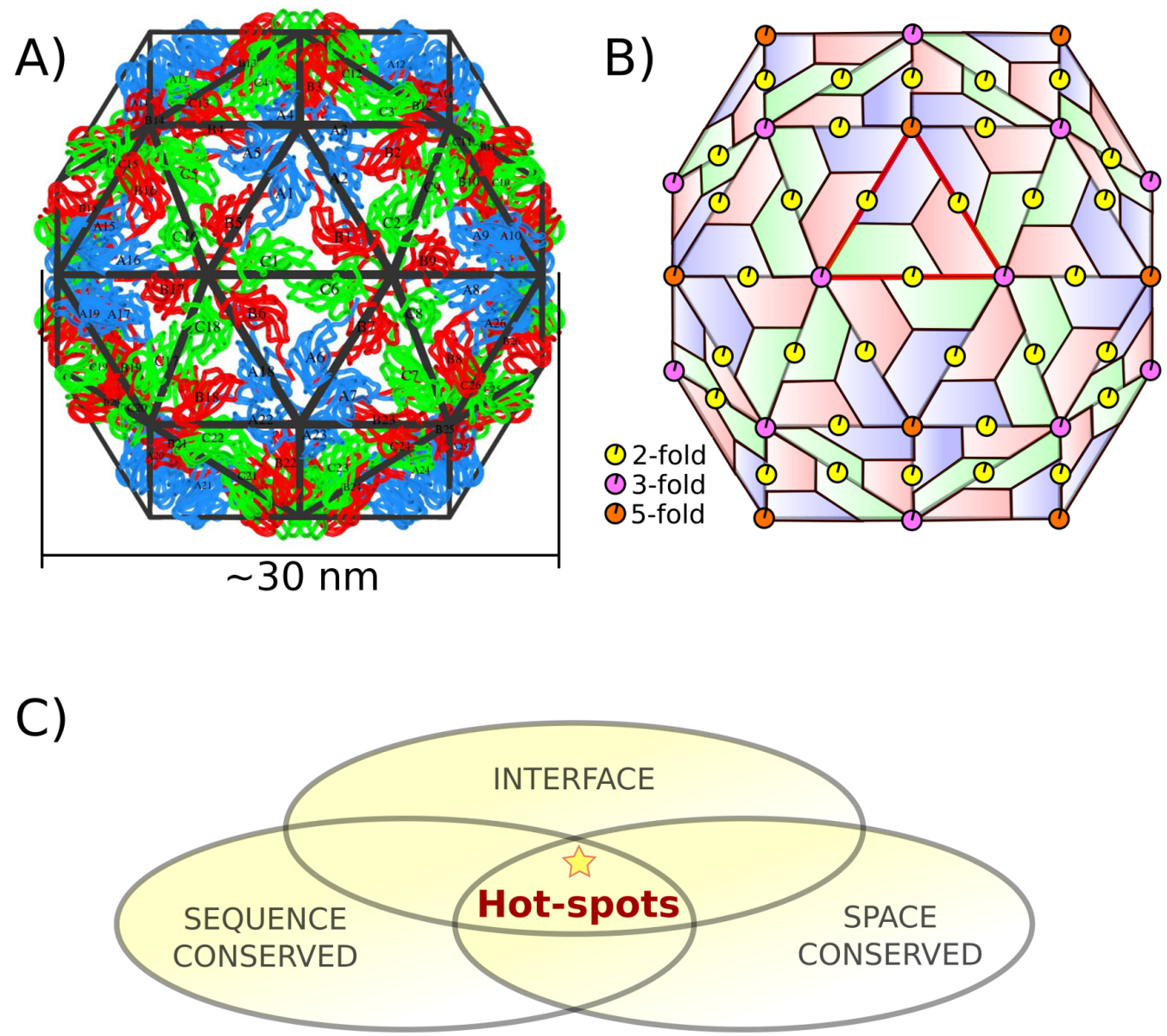

Figure 1. Quaternary structure of the capsid of an icosahedral virus. (A) An example of a $\mathrm{T}=3$ capsid (CCMV), which is formed by the assembly of 180 identical chemically identical proteins (CPs), or subunits, identified here by distinct labels $\left(A_{i}, B_{i}\right.$, and $\left.C_{i}\right)$. The corresponding color represents local arrangement, i.e., subunits of the same color have equivalent locations in the capsid. Each CP interacts and forms interfaces will all its surrounding neighbors. The conceptual icosahedral lattice is shown in black. (B) Each trapezoid represents a CP, or subunit, in the icosahedral arrangement. The location of the symmetry axes that define such geometry are shown. This architecture is characterized by having 60 equivalent triangular faces. One of them is highlighted (red), commonly referred to as the central icosahedral asymmetric unit. (C) Definition of the structure-conserved interface hot spots.

Second, we tested our hypothesis by calculating the binding free energy change produced to the wild type complex when mutating each one of the predicted hot spots. We implemented a rigorous all-atom physical framework with explicit solvent and controlled thermodynamic conditions. Steered Molecular Dynamics (SMD) and Umbrella Sampling simulations were carried out to calculate the value of the binding free energy through the Potential of Mean Force (PMF). Our results show that the structure-conserved interface residues hypothesized to be hot spots do have a significant contribution to the complex formation, as opposed to other nonconserved interface residues. 


\section{Results}

\subsection{Hot Spot Prediction}

\subsubsection{Sequence Conservation of Interface Residues}

We performed an MSA on the CPs of the Bromoviridae family members found in VIPERdb at the time of this writing, namely, Cowpea Chlorotic Mottle Virus (CCMV), Cucumber Mosaic Virus (CMV), Brome Mosaic Virus (BMV), and Tomato Aspermy Virus (TAV) (Appendix A, Figure A2). We corroborated the MSA by a tertiary structure alignment consensus made between the CP of the four viruses [19]. The CPs tertiary structure has a high degree of similarity between the four family members (Figure A3). However, sequence identity is low, suggesting a possible evolutionary structural convergence, as we had previously noted [20]. Sequence conservation is less than $10 \%$ of the total residues. Only half of the family sequence conserved residues are located in an interface: P99, F120, Y159, H172, E176, R179, P188, and V189 (CCMV sequence numbering).

\subsubsection{Space Conservation of Interface Residues}

The multiple quaternary structure alignment of the capsid of the four viruses was achieved through the CapsidMaps methodology [17]. A 2D depiction of the 3D position of the interface residues in the icosahedral asymmetric unit is built by projecting their space coordinates on a plane. Then, a conversion from Cartesian to Spherical coordinates is made. The $\phi-\psi$ angle space is used to generate a two-dimensional map of the quaternary patterns formed by all the interface residues in a particular capsid. The individual CapsidMaps of the four viruses studied in this work are shown in Figure A4. Conserved quaternary positions are readily identified when two or more CapsidMaps are compared. In the case of the Bromoviridae family, only six out of the eight interface sequence conserved residues are also conserved in quaternary structure.

\subsubsection{Hot Spot Predictions by the Structural Conservation Method}

The conservation-based criteria identified a set of six residues, namely, P99, F120, E176, R179, P188, and V189 as hot spots (CCMV sequence numbering). Using the CCMV as a representative member, Figure A5 shows the spatial position of those six residues on a CapsidMap. To locate the specific CP-CP interfaces in the quaternary structure of the capsid where each hot spot is involved, we used VIPERdb's contact tool (Virus Info Page-Annotations-Contact Tables-Which interfaces include a specific residue). Structure-conserved hot spots E176, R179, P188, and V189 were located in the interfaces made around a 2-fold axis, e.g., between subunits A1-B5, A2-B1, C1-C6, C2-C9, and so on. On the other hand, structure-conserved hot spots P99 and F120 were located in the interfaces made around a 3-fold or a 5-fold axis, e.g., between subunits A1-A2, B1-C2, B1-C6, B5-C1, and so on. The relationship between the subunit interfaces and the capsid's symmetry folds, along with the location of the six structure-conserved hot spots of the Bromoviridae family is illustrated in Figure 2. Any dimer related by the same type of symmetry fold in the capsid quaternary structure is equivalent. Given that there is ample evidence that the 2-fold-related dimers, e.g., A2-B1, are the first step in the kinetics of capsid assembly in the case of CCMV [21] (i.e., dimers, pentamers of dimers, hexamers of dimers, etc.), we focused our efforts in the study of this particular type of CP-CP interface in this work.

2.1.4. Hot Spot Predictions by Averaged Energy-Based Alanine Scanning Mutagenesis Approximation Methods

To compare our methodology to other strategies, we used the averaged energy-based alanine scanning computational mutagenesis methods implemented in the ROBBETA, FoldX, SpotOn, and iPPHOT online tools to scan the interface of the 2-fold-related A2-B1 dimer of CCMV. The SpotOn tool only reports a list of potential hot spots (Figure A6). The iPPHOT tool was not able to find any hot spots on the A2-B1 protein complex (Figure A7). ROBBETTA and FoldX both report an approximation 
to the $\Delta \Delta \mathrm{G}$ value in arbitrary units by interface residue. A comparison between all hot spot prediction results is presented in Figure 3. According to the averaged energy-based alanine scanning analyses, there is a consensus on the nonconserved interface residue F186 (Figure A2). In such an approximation, residue F186 shows a larger energy contribution than the rest of the interface residues, including the structure-conserved hot spots predicted with our methodology. Given this result, we included residue F186 in the following rigorous thermodynamic analysis. Furthermore, we randomly selected a nonconserved interface residue (E77) on the CCMV 2-fold-related dimer as an experimental control. The locations of all the residues analyzed in this work in the quaternary structure of the dimer are shown in Figure 4.
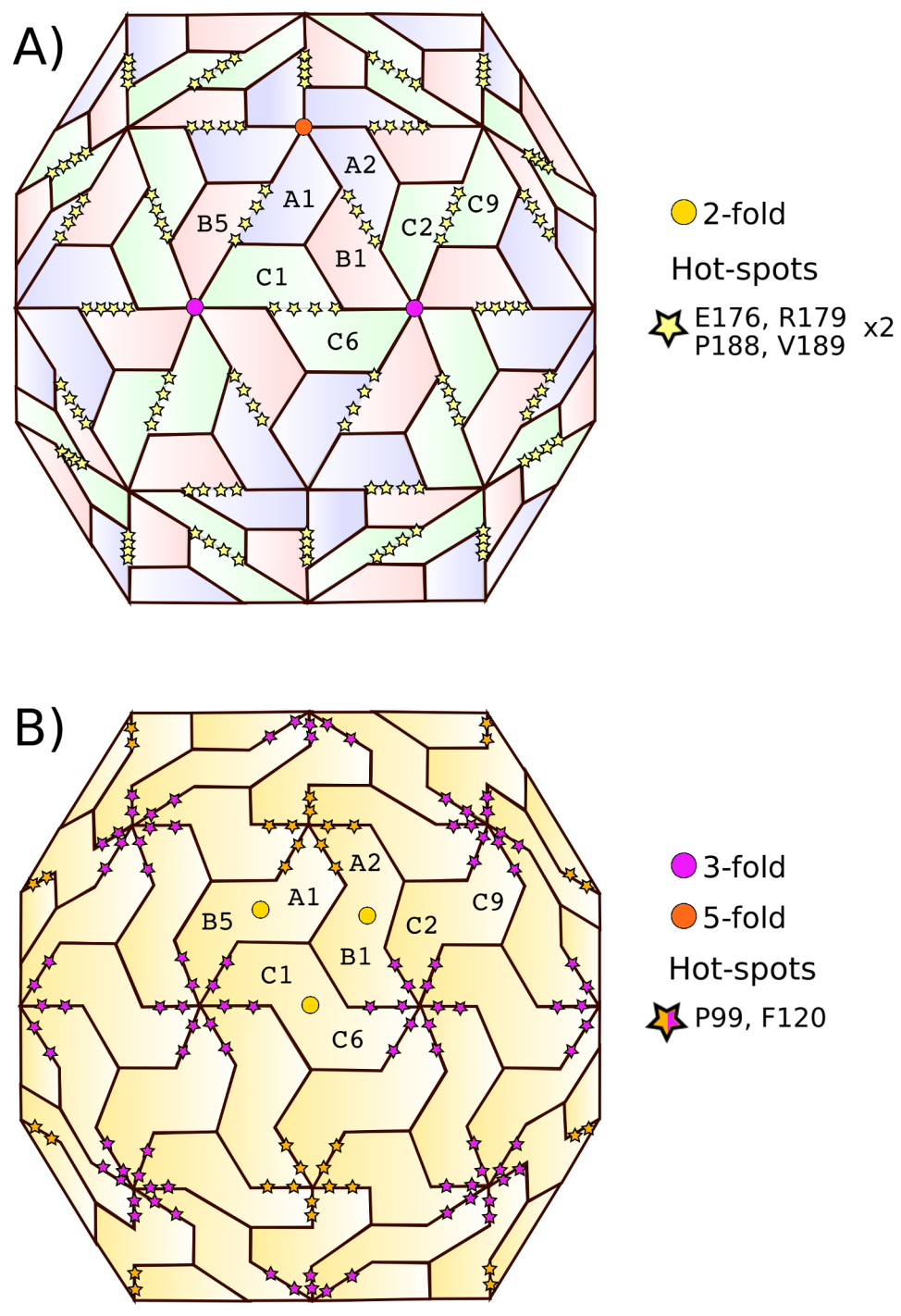

Figure 2. Mapping of the CCMV structure-conserved hot spots on the quaternary structure of the capsid. All hot spots were found to be closely related to a given symmetry axis: (A) 2-fold (E176, R179, P188, and V189) or (B) 3- and 5-fold (P99 and F120). Here, both representations of the capsid's quaternary structure are equivalent, but (B) stresses the fact that the 2-fold-related dimers are the first oligomers formed on the kinetics of capsid self-assembly [21]. Symmetry axes are indicated for the central icosahedral asymmetric unit; only a few subunits are labeled. 


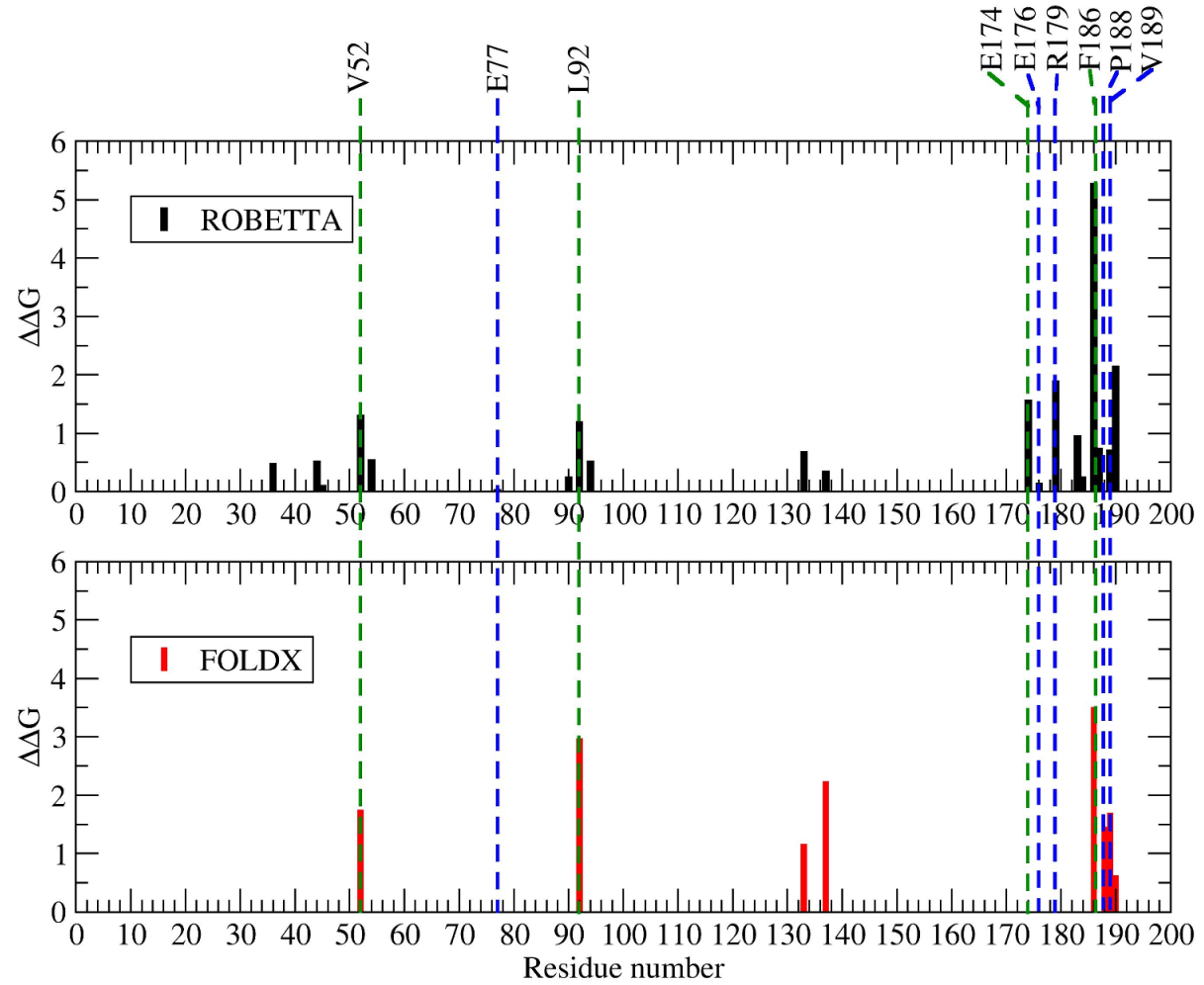

Figure 3. Computational energy-based alanine-scanning mutagenesis results from ROBBETA [5] (black), FoldX [6] (red), and hot spot predictions from SpotOn [7] (green) and this work (blue) of a 2-fold-related dimer interface. Interface residue E77 was randomly selected as an experimental control.
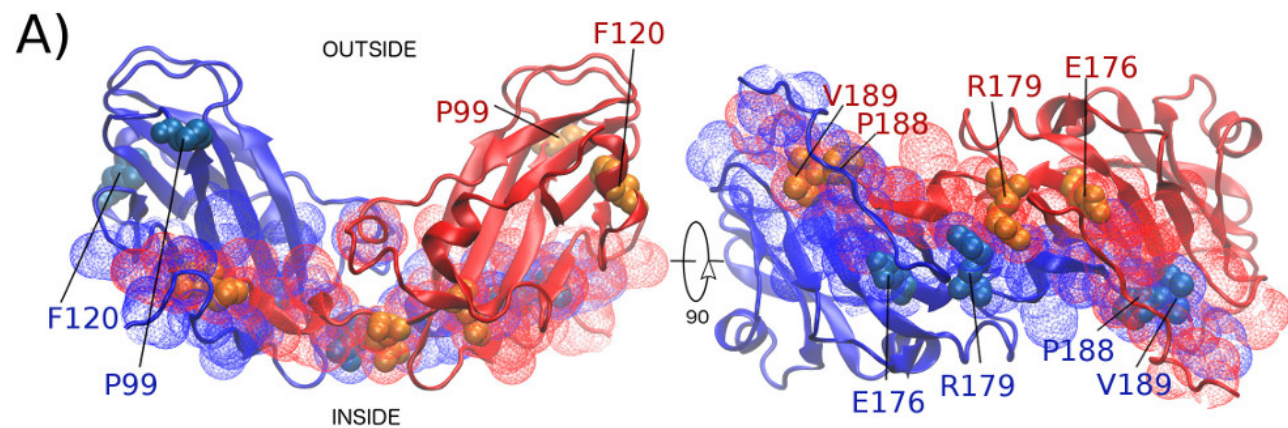

B)

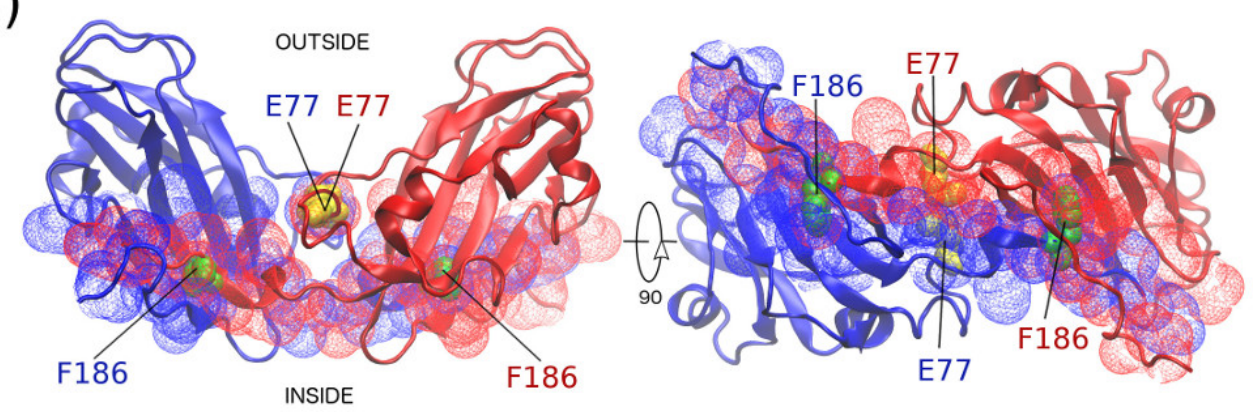

Figure 4. CCMV 2-fold-related dimer. (A) Location of the six structure-conserved hot spots in the quaternary structure (space-fill representation), showing subunit A2 in blue and subunit B1 in red (cartoon representation). The dimer interface residues are shown in mesh representation. A 90-degree rotation has been applied on the right to appreciate the interface better. (B) Location of the interface residues in the control group (using the same representations as before). 
Note that a comparison of several physico-chemical characteristics shows that residue F186 stands out by having a low solvent accessible surface area (SASA) [22], large buried surface area (BSA) [22], lower association energy (AEne) [23] and solvation energy (SolvEne) [24], as well as a larger number of close intermolecular contacts (NumInt) with respect to the structure-conserved hot spots (Table 1). The fact that physico-chemical characteristics are the kind of averaged values used in the alanine-scanning strategies to approximately estimate $\Delta \Delta \mathrm{G}$ is is probably the reason why all the averaged energy-based prediction tools pointed it out.

Table 1. Approximation values of physico-chemical characteristics of the CCMV capsid hot spots. Solvent Accessible Surface Area $\left(\AA^{2}\right)$, Association Energy ( $\left.\mathrm{kcal} / \mathrm{mol}\right)$, Solvation Energy ( $\left.\mathrm{kcal} / \mathrm{mol}\right)$, Buried Surface Area $\left(\AA^{2}\right)$, and number of intermolecular distance-based close contacts.

\begin{tabular}{|c|c|c|c|c|c|}
\hline Residue & SASA & AEne & SolvEne & BSA & NumInt \\
\hline Proline P99 ${ }^{a}$ & 9.70 & -3.29 & -2.03 & 141.20 & 5 \\
\hline Phenylalanine F120 ${ }^{a}$ & 11.10 & -0.73 & -0.35 & 34.73 & 1 \\
\hline Glutamic Acid E176 $b$ & 0.00 & -0.16 & -0.10 & 6.74 & 1 \\
\hline Arginine R179 ${ }^{b}$ & 64.50 & -2.80 & 0.32 & 138.64 & 3 \\
\hline Proline P188 ${ }^{b}$ & 39.20 & -5.03 & -3.47 & 203.97 & 1 \\
\hline Valine V189 ${ }^{b}$ & 28.70 & -4.48 & -2.92 & 187.98 & 4 \\
\hline Glutamic Acid E77 ${ }^{c}$ & 65.50 & -2.72 & -0.54 & 112.78 & 1 \\
\hline Phenylalanine F186 ${ }^{c}$ & 1.70 & -9.58 & -6.70 & 385.04 & 7 \\
\hline
\end{tabular}

\subsection{Hot Spot Validation Through a Rigorous Physical Framework}

Alanine-scanning based strategies are limited by the implicit approximations and physical assumptions they are based on, which are used to make faster calculations. However, their predictions might not be accurate. The methodology we implemented to validate the hot spot predictions through the $\mathrm{CP}-\mathrm{CP}$ binding free energy ( $\Delta \mathrm{G}$ is calculated with Umbrella sampling; PMF; and explicit solvent, temperature, and pressure control), although remarkably computationally more expensive, is a better approximation because it explicitly takes into consideration all interactions and thermodynamic effects present in the studied system. Therefore, the $\Delta G$ values obtained in such a rigorous theoretical framework are more reliable. The Molecular Dynamics trajectory data of all systems studied here can be accessed and visualized at the MDdb Science Gateway at http:/ / www.md-db.org with Study ID 690002.

We mutated each structure-conserved hot spot and control set independently, producing seven variants. The rationale followed in the point mutations was to neutralize charges, change from nonpolar to polar, or from big to small side chain, to disrupt all possible wild type interactions. The final set was E176Q, R179Q, P188A, V189N, F186A, E77Q. A rigorous Molecular Dynamics analysis was performed on systems in thermodynamic equilibrium (NPT), where the proteins were completely solvated with explicit bulk water and $\mathrm{NaCl}$ at room temperature and pressure of $1 \mathrm{~atm}$ (Figure A8). The Umbrella methodology requires to sample conformations spaced along a reaction coordinate, which, in this case, was the distance between the center of mass (COM) of each subunit in the dimer. Therefore, SMD trajectories were generated for the wild type and the set of point mutations, starting from the homodimer complex and pulling the subunits away until their COMs were $10 \mathrm{~nm}$ apart. MD simulations showed that none of the point mutations disrupts the secondary nor the tertiary structure of the CCMV CP (Figures A9 and A10, data available at MDdb). Furthermore, except for mutant $\mathrm{R} 179 \mathrm{Q}$, there is no change with respect to the wild type in the force needed to disassemble the protein complex (Figure A10).

The potential of mean force (PMF), as a function of subunit separation for the seven CCMV CP variants, was built from the Umbrella sampling and the Weighted Histogram Analysis Method (WHAM). The results are shown in Figure 5. The computational cost was 17,500 CPU hours, on average, 
for each one of the seven variants analyzed, totaling on an equivalent of $14 \mathrm{CPU}$ years. As expected, the $\mathrm{CP}-\mathrm{CP}$ distance in which the homodimer complex is thermodynamically stable corresponds to the global minimum in the PMF profile in all cases. Pulling the two subunits $10 \mathrm{~nm}$ away was enough to decrease their interaction energy to zero. This condition is sufficient to confidently assign the global minimum in the PMF profile as the binding free energy in each case.
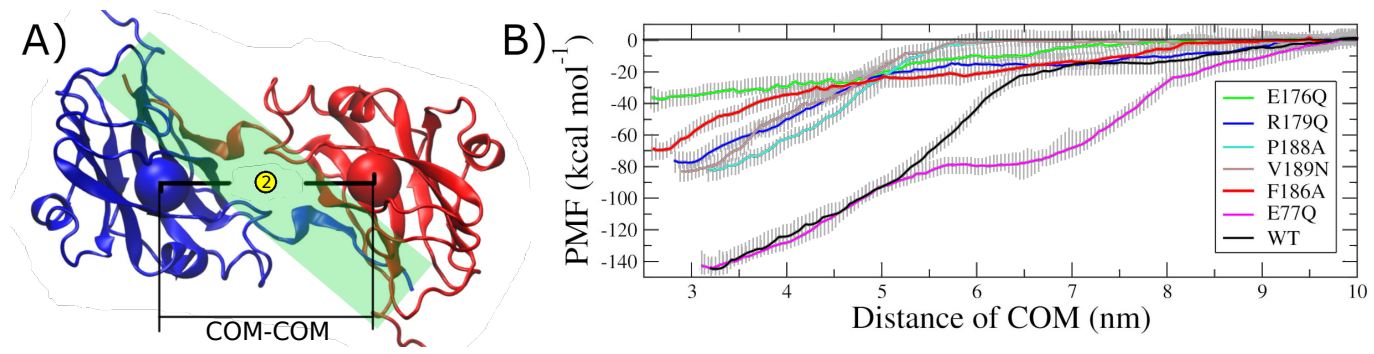

Figure 5. Potential of mean force (PMF) for the seven variants of the CCMV 2-fold-related dimer. (A) Quaternary structure of a 2-fold-related dimer (one subunit in blue and the other in red, cartoon representation). The reaction coordinate to generate the PMF profile was the distance between the center of mass (COM) of each subunit (spheres). The interface region is shaded. (B) PMF profiles as a function of subunit COM-COM distance for the wild type (WT), the point mutations in four structure-conserved hot spots (E176, R179, P188, and V189), and the two residues in the control group (E77 and F186).

Therefore, the change in the dimer interaction due to a point mutation relative to the wild type, $\Delta \Delta \mathrm{G}$, is the difference found between them. Table 2 shows the $\Delta \Delta \mathrm{G}$ values for all the variants studied here. The randomly chosen nonconserved interface residue E77 (experimental control) produces a positive change of less than $1 \mathrm{kcal} / \mathrm{mol}$ when mutated. On the other hand, structure-conserved hot spot E176 produces a positive change of $108 \mathrm{kcal} / \mathrm{mol}$. This value is close to $75 \%$ of the wild type binding free energy. The other three structure-conserved hot spots also produce a positive change, decreasing the thermodynamic stability of the 2 -fold-related dimer by $\sim 45 \%$. Interface residue F186, although not a structure-conserved hot spot, produces a positive change close to $50 \%$.

Table 2. Free energy of dimerization, $\Delta \mathrm{G}$, and change in the dimer interaction due to a point mutation, $\Delta \Delta \mathrm{G}$, relative to the wild type (WT) variant. All units in $(\mathrm{kcal} / \mathrm{mol})$.

\begin{tabular}{cccccc}
\hline Variant & Property & Mutant & Property & $\Delta \mathbf{G}$ & $\boldsymbol{\Delta} \mathbf{\Delta G}$ \\
\hline WT & & & $-144.9 \pm 4.9$ & 0.0 \\
\hline Glutamic Acid E176 $^{a}$ & Negative Charge & Glutamine Q & Neutral & $-37.4 \pm 5.3$ & 107.6 \\
Arginine R179 & Positive Charge & Glutamine Q & Neutral & $-77.5 \pm 6.1$ & 67.5 \\
Proline P188 & Special case & Alanine A & Small & $-82.0 \pm 5.8$ & 63.0 \\
Valine V189 $^{a}$ & Nonpolar & Asparagine N & Polar & $-83.2 \pm 5.6$ & 61.8 \\
\hline Glutamic Acid E77 $^{b}$ & Negative Charge & Glutamine Q & Neutral & $-144.1 \pm 5.8$ & 0.9 \\
Phenylalanine F186 $^{b}$ & Big & Alanine A & Small & $-69.6 \pm 5.9$ & 75.4 \\
\hline
\end{tabular}

${ }^{a}$ 2-fold interactions. ${ }^{b}$ Control group.

\section{Discussion}

In this work, we present a conservation-based strategy to identify protein-protein interface residues potentially relevant to the self-assembly of protein complexes, in particular, icosahedral viral capsids. Our findings provide evidence that a perturbation on any sequence-and-space conserved interface residue decreases the thermodynamic stability of the protein complex, in contrast to other nonconserved interface residues. Seemingly, such structure-conserved hot spots are important on the quaternary level, but not necessarily on the tertiary level. This statement might imply that a mutation 
on any of those conserved residues will not disrupt the protein fold but could prevent the $\mathrm{CP}-\mathrm{CP}$ complex from forming.

On a previous study, we noted the existence of sequence-and-space conserved interface residues in the Nodaviridae family [16]. As in the case of the Bromoviridae family, the location of the conserved interface residues in the quaternary structure of the capsid was not randomly dispersed throughout the $\mathrm{CP}-\mathrm{CP}$ interfaces, but forming patterns around the capsid's symmetry axes. These findings are concomitant with the commonly accepted view of the capsid assembly kinetics. It has been shown that a nucleation seed needs to be formed to start the assembly process. At least in the case of the CCMV, this nucleation seed appears to be a pentamer of 2-fold-related dimers (POD) [25].

Hot spot identification is difficult because there is no apparent correlation between residue type or protein-protein interface composition with the way the complex is formed or the relative orientation between subunits (see Appedix A.1). Our hot spot prediction methodology is straightforward. Even though it is not based on an averaged energy calculation, we have shown, through the PMF, that the structure-conserved interface residues do have a substantial energy contribution to the stability of the protein complex in comparison to nonconserved residues. The fact that an interface residue has been conserved in sequence and space in the quaternary structure during evolution can be explained if that particular residue plays a crucial role in the molecular mechanism of self-assembly, either to direct the process or to provide a stabilization anchoring point between the interacting proteins. Therefore, structural conservation should be a better search criterion than, for example, averaged physico-chemical quantities or the number of intermolecular contacts (e.g., E176 vs. F186, Table 1).

We found that alanine-scanning- and structural conservation-based methodologies give different predictions in the case of CCMV of the Bromoviridae family. Residue F186 is not conserved, therefore it was not accounted for by the conservation-based method. On the other hand, none of the alanine-scanning predictors identified conserved residue E176 as a hot spot. The PMF of residue F186 shows a decrease of 50\% in the binding free energy when mutated. However, the PMF of residue E176 shows a decrease of $75 \%$. Through a fare comparison in this rigorous physical framework, E176 is clearly the most important hot spot residue, but it is missing from all other averaged energy-based prediction tools.

Currently, the averaged energy-based alanine-scanning strategy is the common way to search for hot spots. However, the energy function used is a rough approximation. This makes it a fast method to estimate an approximation to the energy contribution of all interface residues to the binding of a protein complex, one by one. However, such approximation will not necessarily provide an accurate description of the molecular interaction. For example, ROBETTA uses an effective energy function, i.e., making physical assumptions and averaging several contributions into one interaction term, to estimate an approximation to the energy change due to mutations to alanine on the isolated static molecular structure of the protein complex. In contrast, the rigorous thermodynamic analysis we implemented to validate the predictions (Umbrella sampling and PMF) employs a detailed description of all the bonded and non-bonded intra- and intermolecular interactions, explicitly taking into account the contribution of the solvent, temperature, and pressure over a length of time. The calculation of the binding free energy (PMF) was performed when the fully solvated homodimer was thermodynamically equilibrated. This is a closer, more reliable representation of in vitro conditions.

To compare both predictions, we used the method implemented in four available averaged energy-based hot spot prediction tools. Three of them identified nonconserved residue F186 as the one with a distinctive energy contribution to the complex. None of them picked out any of the six structure-conserved hot spots identified by the structure-based methodology. The fact that we found different predictions is not surprising since the fundamental hot spot search criteria used are not the same. Nonetheless, all methodologies (average-energy- or structure conservation-based) were in agreement concerning the interface residue E77 used as a positive control, whose PMF showed a contribution to the binding free energy close to zero (Figure 3). 
The conservation-based prediction methodology has the limitation that a single structure is not enough to detect interface residues conserved at the quaternary level, i.e., the algorithm requires as many quaternary structures as possible. If a rigorous thermodynamic validation is required, the computation of the binding free energy (PMF) is costly and requires the use of high-performance computing. Nonetheless, that might not represent a problem nowadays with the increased access to supercomputing resources. All the computational packages used in our validation analysis are free and open source.

The hot spot prediction in silico thermodynamic validation by the binding free energy (PMF) shows that the structure-conserved interface residues do have a large contribution to the formation and stability of the protein complex. That result confirms our hypothesis; however, results of an in vitro biochemical analysis and biophysical validation are reported in a separate report. In view of our findings, a similar study of the thermodynamic contribution of structure-conserved hot spots P99 and F120 is in progress. Those residues were excluded from this work because they are involved in CP-CP interfaces different to the 2-fold-related. Most likely, their role will be in the formation of intermediate states in the process of capsid assembly, e.g., PODs.

\section{Materials and Methods}

\subsection{Multiple Sequence Alignment}

The Geneious R7 software was used to carry out a multiple sequence alignment (MSA) on a personal computer. The amino acid sequence of the $\mathrm{CP}$ of members of the Bromoviridae family whose molecular structure was available in the VIPERdb Science Gateway [18] were included.

\subsection{Interface Residues and Quaternary Structure Alignment}

We used the CapsidMaps [17] tool of VIPERdb to find the interface residues of members of the Bromoviridae family. The intrafamily structural alignments were carried out using the method previously described [16]. The cured crystallographic structures of the viruses were queried to produce a CapsidMap of the interface residues. The $\phi$ and $\psi$ coordinates of each residue were recorded and then compared between viruses. An overlap threshold of $3^{\circ}$ in both angles was allowed to consider a conserved residue position in space.

\subsection{Hot Spot In Silico Mutations}

The 3D structure of the wild type (WT) 2-fold-related dimer was obtained from the Oligomer Generator tool of VIPERdb. Indications were followed, and the necessary parameters were introduced to obtain a file in PDB format with the atomic coordinates of the selected dimer (A2, B1). All hydrogen atoms were added with the WHAT IF Web Interface. The protonation state of histidine residues was set to physiological conditions ( $\mathrm{pH}$ 7.0). Then, the Mutator Plugin tool of the Visual Molecular Dynamics software (VMD) [26] was used to generate one dimer with a point mutation for each one of the residues resulting from the structural conservation prediction. An additional point mutation was randomly selected from the A-B interface residues as an experimental control (E77).

\subsection{Alanine Scanning Mutagenesis}

A comparison with averaged energy-based hot spot prediction tools was made. We used the alanine scanning mutagenesis computational methods implemented in the ROBBETA [5], FoldX [6], SpotOn [7], and iPPHOT [8] (alignment created by ConSurf [27] using UNIREF90 and MAFFT) online tools. These are fast but coarse approaches for the prediction of energetically relevant amino acid residues in protein-protein interfaces. In all cases, the input was the 3D structure of the WT dimer in PDB format. The result was a list of residues predicted to significantly destabilize the interface when mutated to alanine, based on an approximated energy function. 


\subsection{Steered Molecular Dynamics}

To accurately calculate the dimers binding free energy with a rigorous physical framework, a previously reported computational method using molecular dynamics was implemented [28]. We followed the same procedure for all the variants produced in the previous section, both in the monomer or dimer oligomerization state. The CHARMM27 all-atom force field (FF) plus CMAP for proteins was used to describe molecular interactions [29]. This FF was chosen because it was shown that substantial deviations from experimental backbone root-mean-square fluctuations and N-H NMR order parameters obtained in the MD trajectories are eliminated by the CMAP correction, therefore improving dynamical and structural properties of proteins. The dimers were solvated with liquid water using the TIP3P potential function [30].

All simulations were performed using the GROMACS 4.5.5 suite [31]. The monomers were placed in a cubic box, whereas the dimers were placed inside a rectangular box (Figure A8). In either case, the dimensions of the simulation box were chosen such that the minimum distance between any atom of the protein and the walls were no less than $1.0 \mathrm{~nm}$, as well as to provide sufficient space for the pulling to take place along the x-axis. The empty volume was filled with water molecules. Also, $\mathrm{Na}^{+}$and $\mathrm{Cl}^{-}$ions were added in a proportion that would neutralize the overall charge of the system and obtain a final salt concentration of $100 \mathrm{mM}$. Bad contacts between any two atoms were removed by energy minimization of the whole system using the steepest descent algorithm with a force tolerance of $100.0 \mathrm{~kJ} / \mathrm{mol} / \mathrm{nm}$ and a 0.01 step size. Isochoric-isothermal (NVT) equilibration of solvent molecules to $300 \mathrm{~K}$ was performed for $100 \mathrm{ps}$, with the proteins heavy atoms being restrained by a harmonic potential with a force constant of $1000.0 \mathrm{~kJ} / \mathrm{mol} / \mathrm{nm}$. A subsequent isobaric-isothermal (NPT) equilibration to adjust the system density was performed for another $100 \mathrm{ps}$ at $1 \mathrm{bar}$.

Further structural equilibration simulations were carried out in the NPT ensemble at a temperature of $300 \mathrm{~K}$ and a pressure of 1 bar for $1 \mathrm{~ns}$ removing all position restraints. The temperature was maintained using the V-rescale thermostat with a coupling time constant of $0.1 \mathrm{ps}$. The dimer and solvent molecules were coupled to separate thermostats to avoid the hot solvent-cold solute issue. The pressure was regulated using the isotropic Parrinello-Rahman barostat with a coupling time of $2.0 \mathrm{ps}$ and compressibility of $4.5 \times 10^{-5} \mathrm{bar}^{-1}$. Bonds involving hydrogen were constrained to their equilibrium values using the LINCS algorithm. The non-bonded interactions (Lennard-Jones and electrostatic) were truncated at $1.0 \mathrm{~nm}$. Long-range electrostatic interactions beyond the cut-off distance were calculated using the particle mesh Ewald (PME) method, with a Fourier spacing of $0.16 \mathrm{~nm}$ and a cubic interpolation of order 4 . A long-range analytic dispersion correction was also applied to both energy and pressure to account for the truncation of the Lennard-Jones interaction. The time-dependent dynamics of the system was evolved using the leap-frog integrator with a time step of 2 fs.

After full equilibration, position restraints were set again for subunit A only; therefore, using it as an immobile reference for the pulling simulations. For each of the dimer variants, subunit $B$ was pulled away from subunit A along the $\mathrm{x}$-axis over $2000 \mathrm{ps}$, using a spring constant of $2000 \mathrm{~kJ} \mathrm{~mol}^{-1} \mathrm{~nm}^{-2}$ and a pull rate of $0.0035 \mathrm{~nm} \mathrm{ps}^{-1}\left(0.035 \AA \mathrm{As}^{-1}\right)$. A final center-of-mass (COM) distance of approximately $7 \mathrm{~nm}$ between subunits $\mathrm{A}$ and $\mathrm{B}$ was achieved.

\subsection{Umbrella Sampling}

From the previous SMD trajectories, snapshots were taken to generate the starting configurations for the Umbrella Sampling windows [32]. An asymmetric distribution of windows was used, such that the spacing was between 1.5 and $2 \mathrm{~nm}$ COM separation. Such spacing resulted in 42 windows per dimer. For each window, $5 \mathrm{~ns}$ of MD was performed for a total simulation time of $210 \mathrm{~ns} \times 7$ dimers utilized for Umbrella Sampling. Analysis of results was performed with the Weighted Histogram Analysis Method (WHAM) [33] for the generation of the Potential of Mean Force (PMF) profile as a function of the reaction coordinate (COM separation). 
Author Contributions: Conceptualization, M.C.-T.; methodology, M.C.-T.; validation, A.D.-V., J.M.F.-G. and M.C.-T.; formal analysis, A.D.-V., J.M.F.-G., and M.C.-T.; investigation, A.D.-V., J.M.F.-G., and M.C.-T.; resources, M.C.-T.; data curation, M.C.-T.; writing-original draft preparation, M.C.-T.; writing—review and editing, M.C.-T.; visualization, A.D.-V., J.M.F.-G., and M.C.-T.; supervision, M.C.-T.; project administration, M.C.-T.; funding acquisition, M.C.-T.

Funding: This research was funded by the Consejo Nacional de Ciencia y Tecnología México (CONACYT grant number 132376) and Fondo Sectorial de Investigación para la Educación (grant number A1-S-17041) to M.C.-T., and Secretaría de Investigación y Posgrado del Instituto Politécnico Nacional (project number 20196271) to J.M.F.-G.

Acknowledgments: All molecular dynamics simulations involved in the mutagenesis analysis reported here were performed with the bmdhpc computing resources of the Biomolecular Diversity Lab (tripplab.com) at CINVESTAV Unidad Monterrey, Mexico, and the computing center Insurgente (www.cimat.mx/en/node/996) at the Centro de Investigación en Matemáticas, Guanajuato, Mexico, thanks to the facilities kindly provided by Salvador Botello-Rionda from the Computer Sciences Department.

Conflicts of Interest: The authors declare no conflicts of interest. The funders had no role in the design of the study; in the collection, analyses, or interpretation of data; in the writing of the manuscript; or in the decision to publish the results.

\section{Abbreviations}

The following abbreviations are used in this manuscript.

$\begin{array}{ll}\text { CCMV } & \text { Cowpea Chlorotic Mottle Virus } \\ \text { CP } & \text { capsid protein } \\ \text { WT } & \text { wild type } \\ \text { COM } & \text { center-of-mass } \\ \text { MD } & \text { Molecular Dynamics } \\ \text { SMD } & \text { Steered Molecular Dynamics }\end{array}$

\section{Appendix A. Supplementary Data}

\section{Appendix A.1. Composition of the 2-Fold-Related Interface}

The Cowpea Chlorotic Mottle Virus (CCMV) belongs to the Bromoviridae family. We use it as a representative member from this point on. The CCMV capsid protein residue composition is not homogeneous. An analysis of the CPs quaternary structure shows this holds for the interfaces too (Figure A1). The structural regions found in a protein, namely, interface, core, and surface, grouped by their physicochemical nature are summarized in Table A1 for the case of the CCMV CP. A proportion of 40-40-20 percentage is found for residues on the interface, surface, and core, respectively. Half the total residues are nonpolar, distributed in 20-20-10 proportion in the same order. Even though there is a couple of cysteines in the core, they are too far apart to form a disulfide bond. The small number of aromatic residues seem to be evenly distributed throughout the protein structure. However, a significantly larger number of charged residues are found in the interface with respect to any other structural region in this particular case.

Other physical properties have been used to characterize a protein. Some of these are the hydrophobicity $(\mathrm{H})$ [34], the solvent accessible surface area (SASA) [22], the association energy (AEne) [23], the solvation energy (SolvEne) [24], and the buried surface area (BSA) [22]. We show a summary of these properties for the structural regions of the CCMV CP in Table A2, as reported on VIPERdb's contact tables [18] (entry ID 1cwp). The total hydrophobicity by structural groups was estimated as the sum of the hydrophobicity index value of all residues in each group. As expected, the core and interface regions present large and equal values of hydrophobicity. Nonetheless, the surface exposed to the solvent also presents a large hydrophobicity. On the other hand, the surface in contact with the nucleic acids is rather hydrophilic. In terms of surface areas, the core region presents low values in both BSA and SASA. The outside surface presents low BSA but high SASA. The interface region has large values both in BSA and SASA. This observation could be related to the local flexibility of each region [35]. It is not surprising that most of the AEne come from the interface region. 


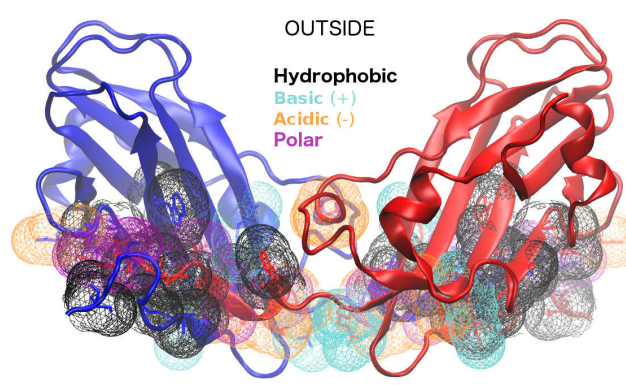

INSIDE

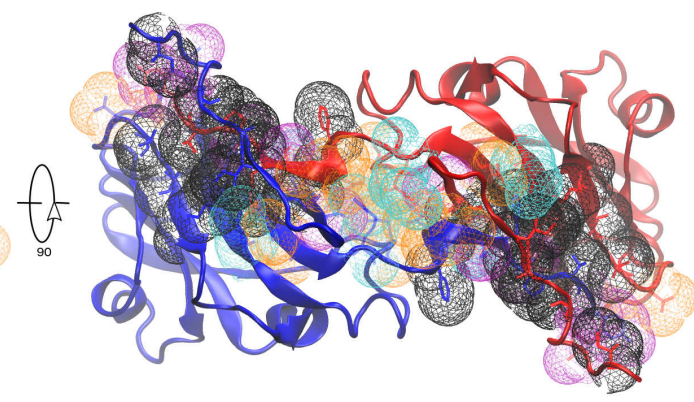

Figure A1. CCMV 2-fold-related homodimer. Front view of the complex of subunit A2 (blue) and subunit B1 (red) in cartoon representation. The outside of the capsid is on top and the interior in the bottom. The dimer interface residues are shown in mesh representation, color-coded to represent the individual physicochemical property (hydrophobic, polar, basic, or acid). A 90-degree rotation has been applied on the right to appreciate the interface better.
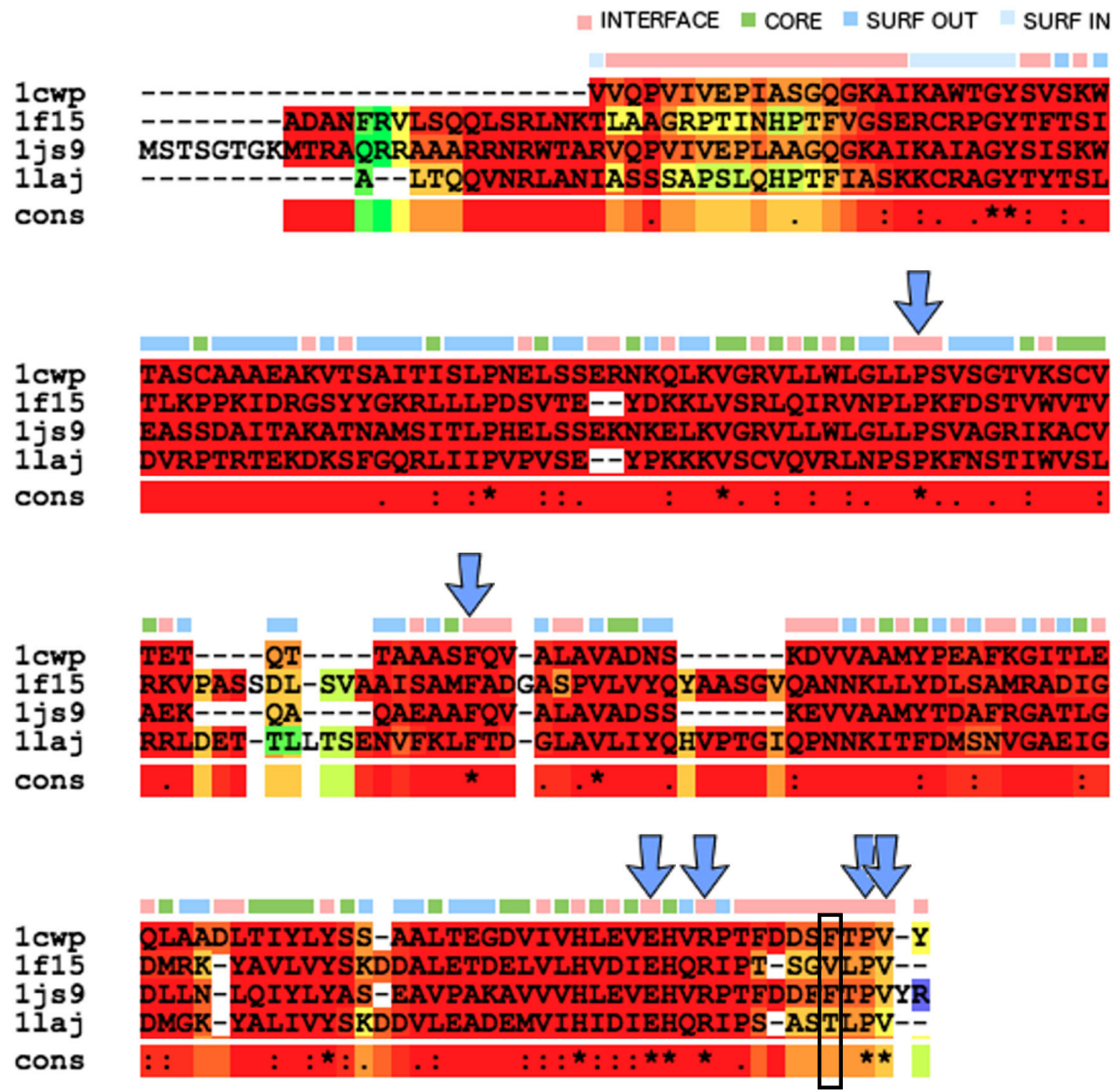

Figure A2. Multiple sequence alignment (MSA) of the Bromoviridae family members found in VIPERdb: Cowpea Chlorotic Mottle Virus (1cwp), Cucumber Mosaic Virus (1f15), Brome Mosaic Virus (1js9), and Tomato Aspermy Virus (1laj). The background color of the MSA represents tertiary structural similarity (low in green, medium in yellow, and high in red). Top row: location of each residue in the capsids quaternary structure, mapping the sequence into four distinct groups (interface: red; core: green: outer surface: blue; or inner surface: light blue-). Hot spots are indicated with arrows; P99, F120, E176, R179, P188, and V189. Position 186 is shown to be nonconserved interface. 
Table A1. CCMV capsid protein residue composition by structural groups. Number of residues is displayed in percentage $(\%)$.

\begin{tabular}{cccccccc}
\hline Group & Nonpolar & Polar & Negative & Positive & Total & CYS & TRP \\
\hline Interface & $33(20)$ & $14(9)$ & $12(8)$ & $10(6)$ & $69(42)$ & & W94 \\
Dimer ${ }^{a}$ & $14(9)$ & $5(3)$ & $5(3)$ & $3(1)$ & $27(16)$ & & W94 \\
Core & $22(13)$ & $8(5)$ & $2(1)$ & $1(1)$ & $33(20)$ & C59, C108 & \\
Surf-Out & $31(19)$ & $20(12)$ & $2(1)$ & $2(1)$ & $55(34)$ & & W55 \\
Surf-In & $4(2)$ & $2(1)$ & $0(0)$ & $1(1)$ & $7(4)$ & W47 \\
\hline Total & $90(54)$ & $44(27)$ & $16(10)$ & $14(9)$ & $164(100)$ \\
\hline \multicolumn{7}{c}{ Counted in Interface residues. }
\end{tabular}

Table A2. CCMV capsid protein physical-chemical properties by structural groups. Hydrophobicity (Mehler scale), Solvent Accesible Surface Area $\left(\AA^{2}\right)$, Association Energy (kcal/mol), Solvation Energy (kcal/mol), and Buried Surface Area $\left(\AA^{2}\right)$.

\begin{tabular}{cccccc}
\hline Group & H & SASA & AEne & SolvEne & BSA \\
\hline Interface & 18.5 & 2382.3 & -147.1 & -51.4 & 6351.9 \\
Dimer $^{a}$ & 8.6 & 864.2 & -87.1 & -34.4 & 3707.0 \\
Core & 19.2 & 78.4 & -0.2 & -0.1 & 8.9 \\
Surf-Out & 14.6 & 2853.5 & -3.4 & -0.8 & 171.1 \\
Surf-In & 2.2 & 604.3 & -2.5 & -1.2 & 118.4 \\
\hline
\end{tabular}

${ }^{a}$ Part of the Interface residues.

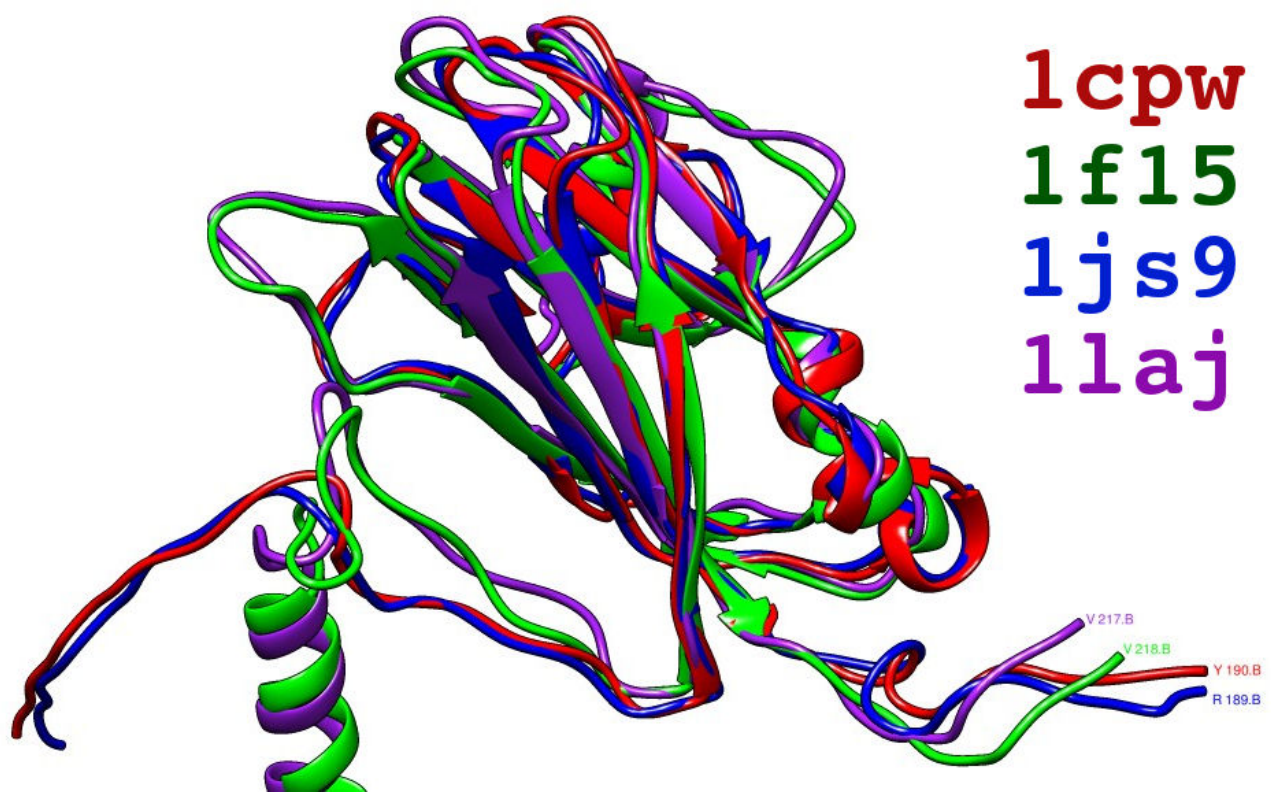

Figure A3. Multiple structure alignment of the $\mathrm{CP}$ of members of the Bromoviridae family: Cowpea Chlorotic Mottle Virus (1cwp in red), Cucumber Mosaic Virus (1f15 in green), Brome Mosaic Virus (1js9 in blue), and Tomato Aspermy Virus (1laj in purple). 

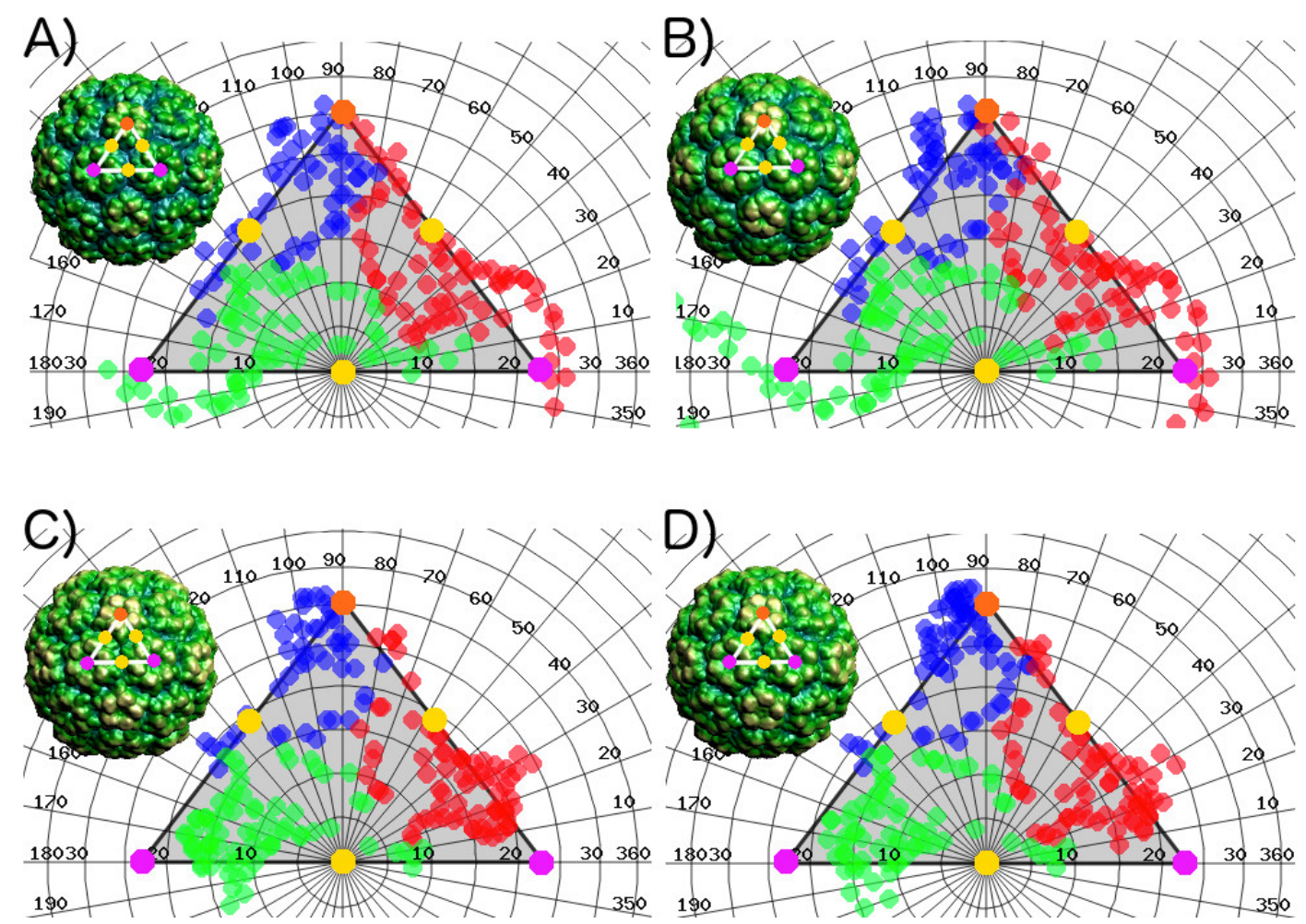

Figure A4. CapsidMaps of the interface residues of members of the Bromoviridae family, namely, (A) Cowpea Chlorotic Mottle Virus, (B) Brome Mosaic Virus, (C) Cucumber Mosaic Virus, and (D) Tomato Aspermy Virus. Each circle represents the location of an interface residue in $\phi-\psi$ space. The color used indicates the subunit to which the residue belongs ( $\mathrm{A}$ in blue, B in red, and $\mathrm{C}$ in green). The icosahedral asymmetric unit used is indicated on the full capsid (insets), showing one 5-fold (orange), two 3-fold (magenta), and three 2-fold (yellow) symmetry axes. The 5-fold corresponds to $\phi=90-\psi=30$, and the opposite 2-fold corresponds to $\phi=\psi=0$.

A)

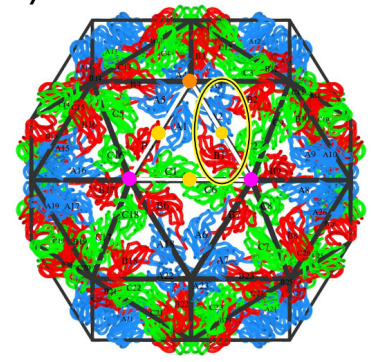

B)

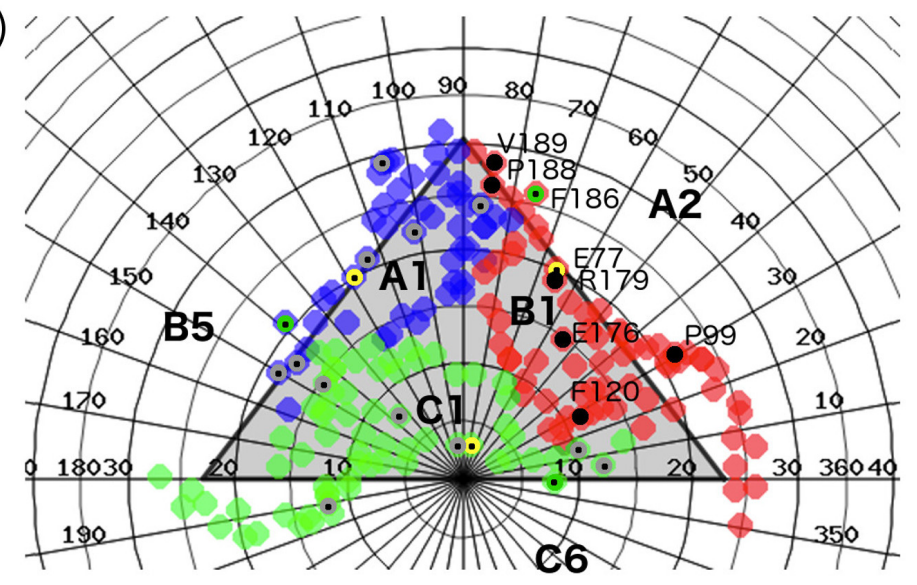

Figure A5. Multiple quaternary structure alignment of the Bromoviridae family members found in VIPERdb. The CCMV is used as the representative model. (A) Full capsid of the CCMV. The icosahedral asymmetric unit used in the analysis is indicated on white, showing one 5-fold (orange), two 3-fold (magenta), and three 2-fold (yellow) symmetry axes. The 5-fold corresponds to $\phi=90-\psi=30$, and the opposite 2-fold corresponds to $\phi=\psi=0$. All dimers related through a 2-fold axis are equivalent. One of them is highlighted, formed by the A2-B1 subunits. (B) CapsidMap of the CCMV interface residues, indicating the location of the hot spots for subunit B1 (black dots), A1 and C1 (gray-black dots) in $\phi-\psi$ space. Location of the interface residues in the control group is also indicated (yellow and green dots). 


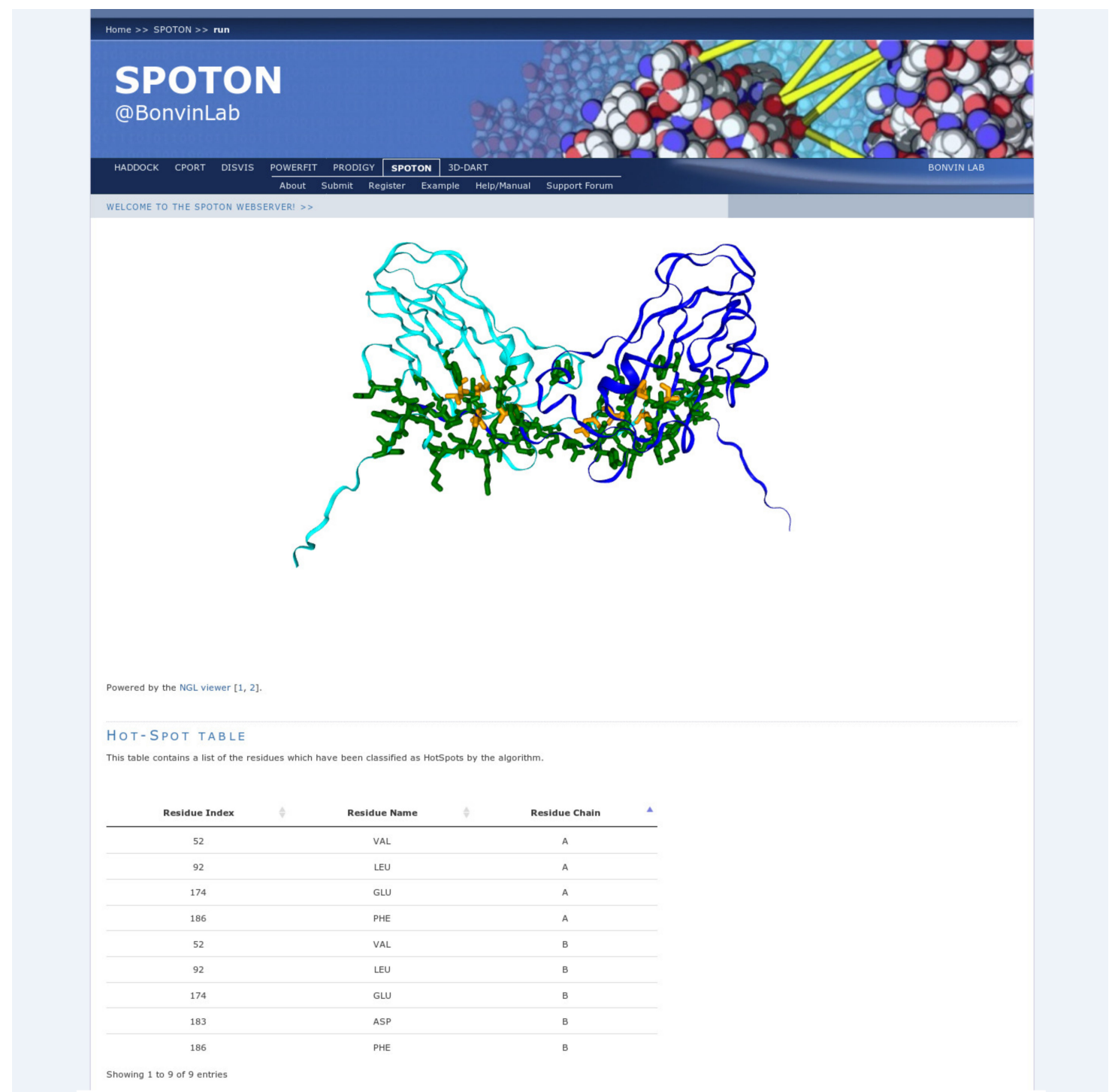

Figure A6. SpotOn [7] hot spot predictions for the structure of a representative 2-fold-related dimer. 


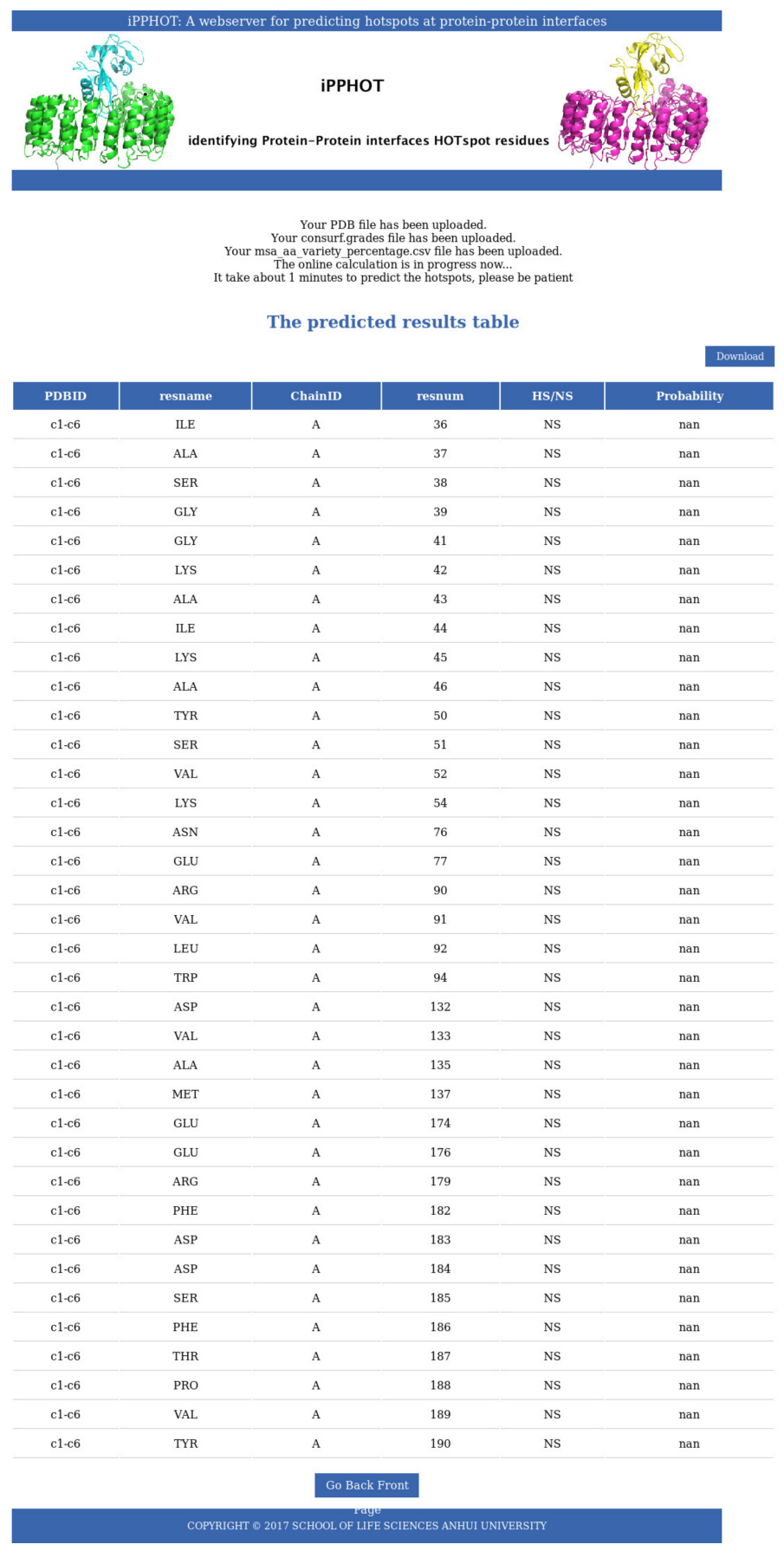

Figure A7. iPPHOT [8] hot spot predictions for the structure of a representative 2-fold-related dimer. All interface residues were predicted to be null-spots (NS). 

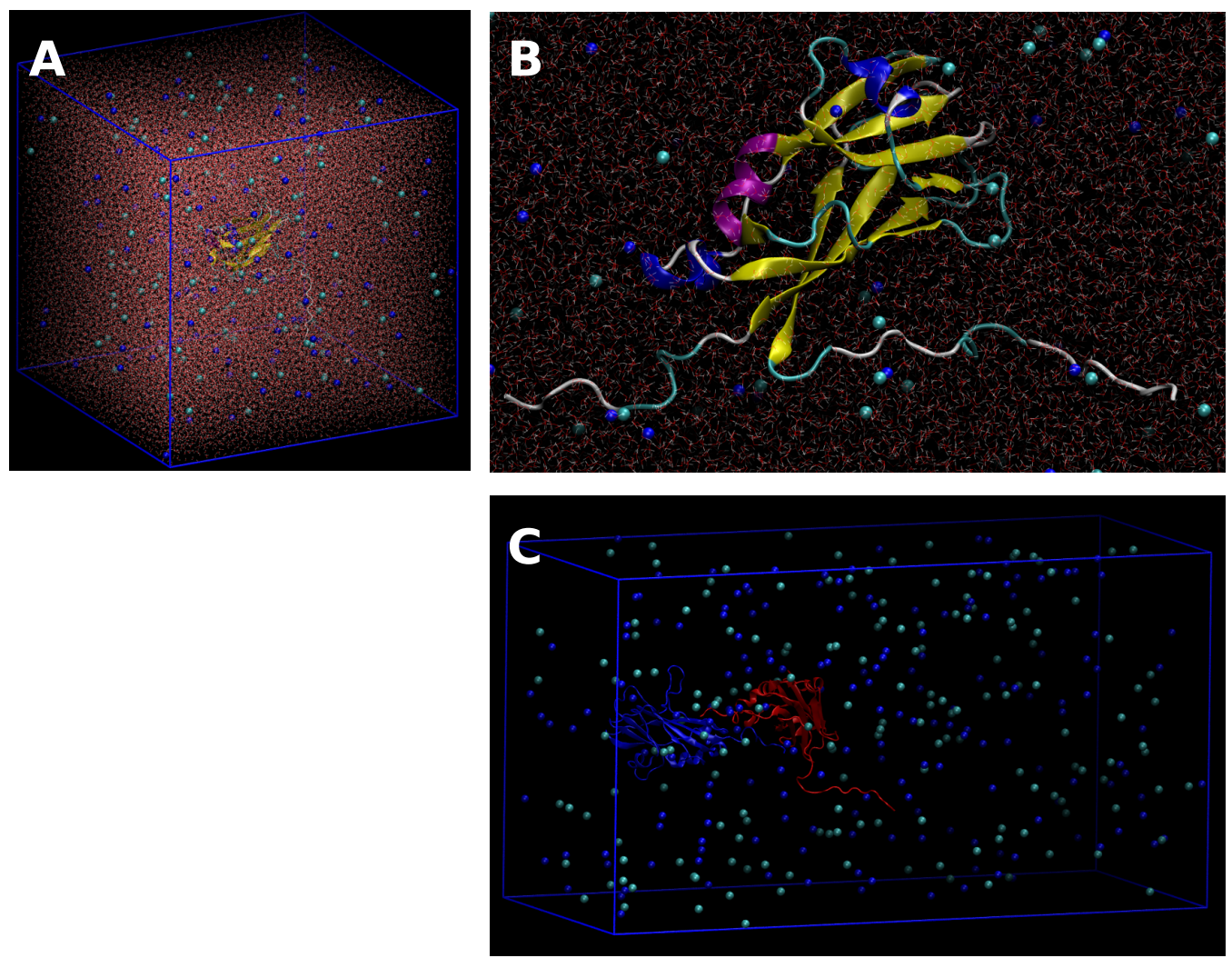

\section{D}

Figure A8. Simulation box of the Molecular Dynamics analysis of the CP of the CCMV in a monomer (A,B) or dimer (C,D) oligomerization state. (A) The CP (cartoon) was solvated with water (lines) and $\mathrm{NaCl}$ (spheres). (B) A closed-up view of the $\mathrm{CP}$ in the aqueous solution. Colors represent secondary structure and type of ion. (C) In the case of Steered Molecular Dynamics, the CP-CP dimer was also solvated with water (not shown) and $\mathrm{NaCl}$ is in a larger box. (D) COM-COM distance based configurations were used in Umbrella Sampling to build the PMF (water and $\mathrm{NaCl}$ not shown). Trajectory data available at MDdb. 

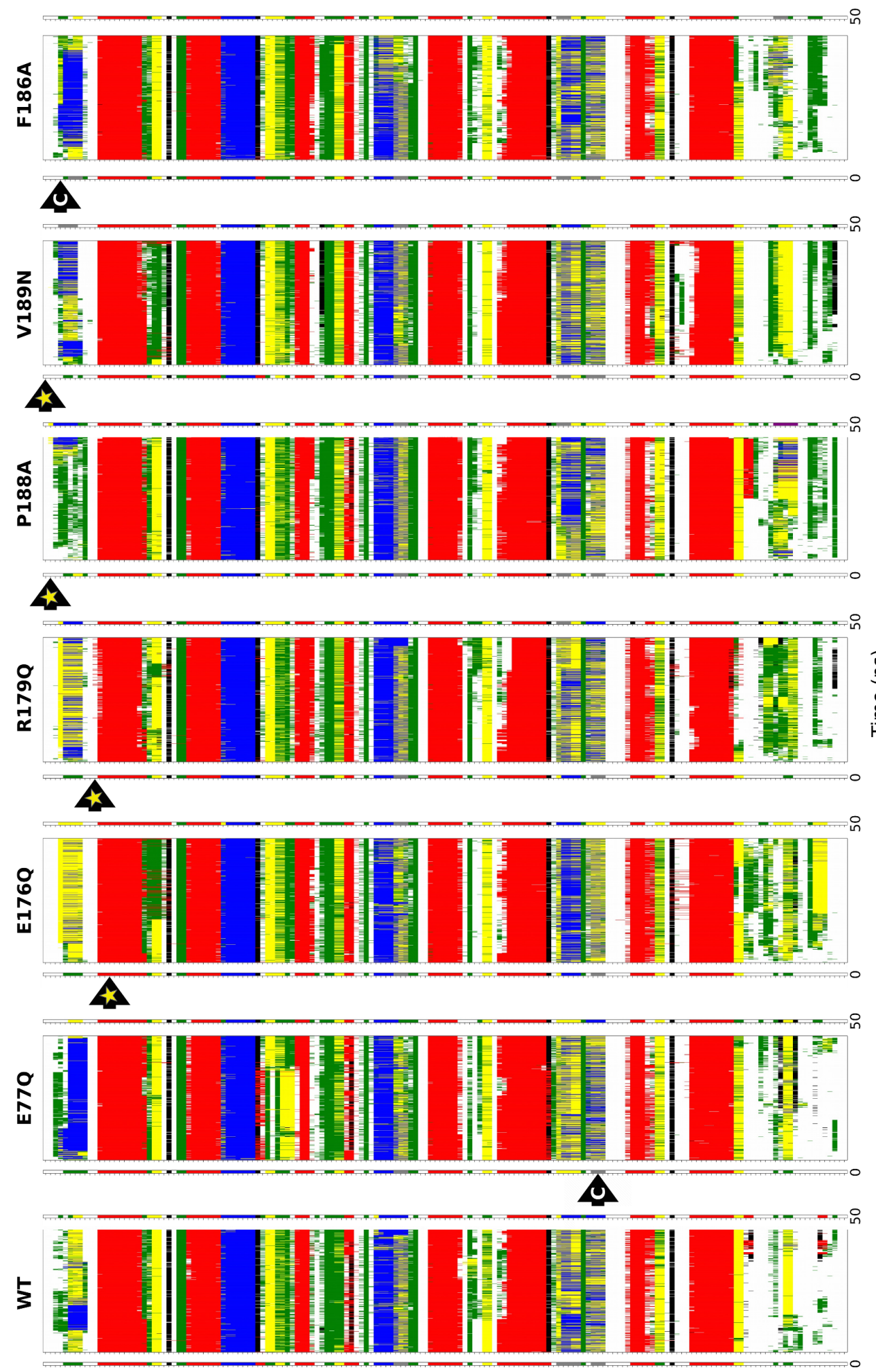

әnp!səy

Figure A9. Analysis of monomer CP secondary structure relaxation by residue number over $50 \mathrm{~ns}$ of a Molecular Dynamics simulations for the seven CCMV CP variants studied in this work: wild type (WT), structure-conserved hot spots (stars), and control group (C). It can be seen that the point mutations (black arrows) do not disrupt the secondary structure of the CP. Trajectory data available at MDdb. 

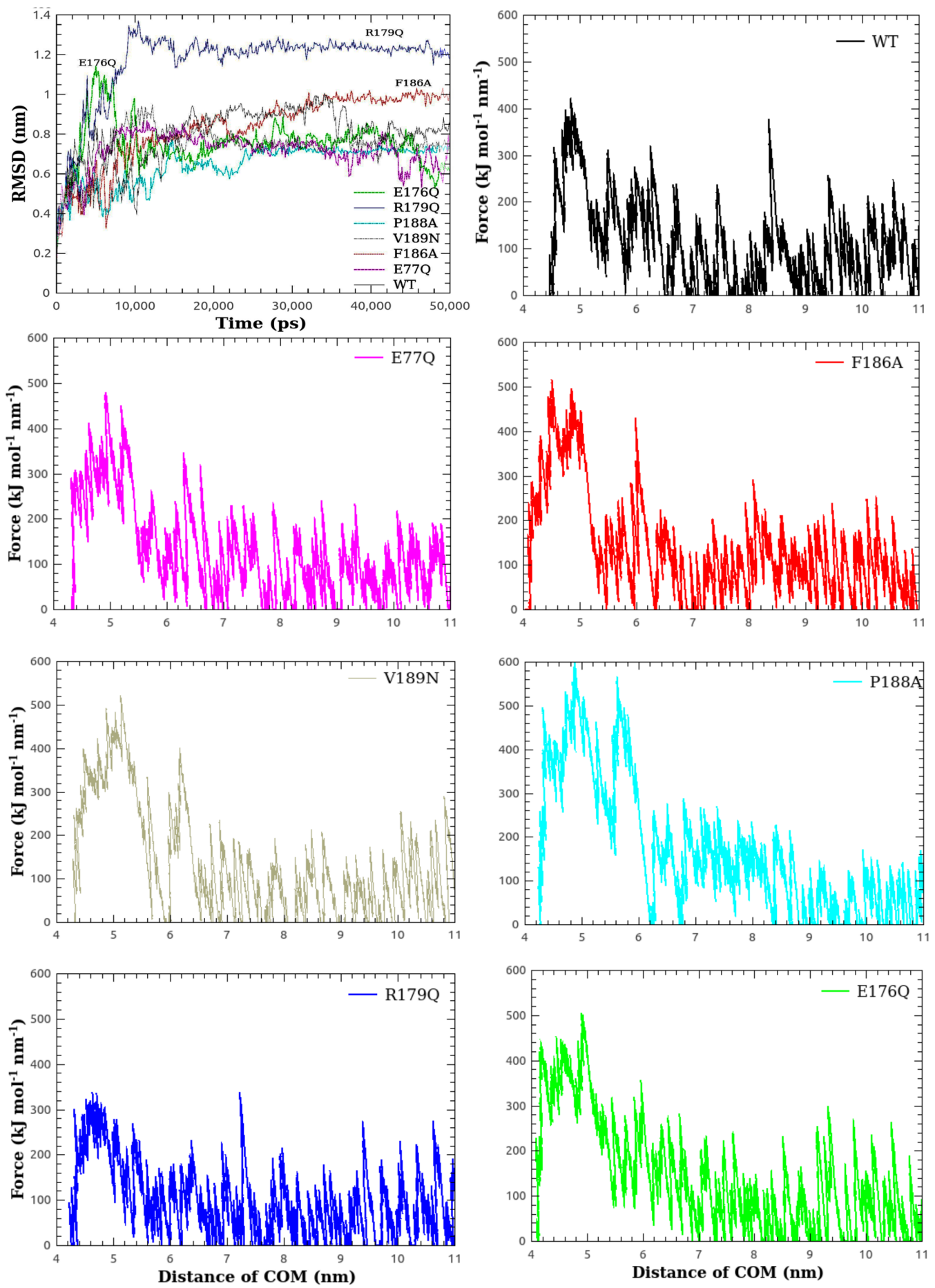

Figure A10. Analysis of monomer CP tertiary structure relaxation over $50 \mathrm{~ns}$ of a Molecular Dynamics simulations (RMSD vs. time) and 2-fold-related dimer breaking force (F vs. COM-COM distance) for the seven CCMV CP variants studied in this work. It can be seen that the point mutations do not disrupt the tertiary structure of the CP. Also, the force needed to break the protein complex remains approximately the same $\left(\approx 400 \mathrm{~kJ} \mathrm{~mol}^{-1} \mathrm{~nm}^{-1}\right)$. Trajectory data available at MDdb. 


\section{References}

1. Twarock, R.; Luque, A. Structural puzzles in virology solved with an overarching icosahedral design principle. Nat. Commun. 2019, 10, 4414. [CrossRef] [PubMed]

2. Castón, J.R.; Carrascosa, J.L. The Basic Architecture of Viruses. In Structure and Physics of Viruses: An Integrated Textbook; Mateu, M.G., Ed.; Springer: Dordrecht, The Netherlands, 2013; pp. 53-75.

3. Crick, F.H.C.; Watson, J.D. Structure of Small Viruses. Nature 1956, 177, 473-475. [CrossRef] [PubMed]

4. Caspar, D.L.D.; Klug, A. Physical Principles in the Construction of Regular Viruses. Cold Spring Harb. Symp. Quant. Biol. 1962, 27, 1-24. [CrossRef] [PubMed]

5. Kortemme, T.; Kim, D.E.; Baker, D. Computational Alanine Scanning of Protein-Protein Interfaces. Sci. STKE 2004, 2004, pl2. [CrossRef] [PubMed]

6. Schymkowitz, J.; Borg, J.; Stricher, F.; Nys, R.; Rousseau, F.; Serrano, L. The FoldX web server: An online force field. Nucleic Acids Res. 2005, 33, W382-W388. [CrossRef]

7. Moreira, I.S.; Koukos, P.I.; Melo, R.; Almeida, J.G.; Preto, A.J.; Schaarschmidt, J.; Trellet, M.; Gümüş, Z.H.; Costa, J.; Bonvin, A.M.J.J. SpotOn: High Accuracy Identification of Protein-Protein Interface Hot Spots. Sci. Rep. 2017, 7, 8007. [CrossRef] [PubMed]

8. Qiao, Y.; Xiong, Y.; Gao, H.; Zhu, X.; Chen, P. Protein-protein interface hot spots prediction based on a hybrid feature selection strategy. BMC Bioinform. 2018, 19, 14. [CrossRef]

9. Assi, S.A.; Tanaka, T.; Rabbitts, T.H.; Fernandez-Fuentes, N. PCRPi: Presaging Critical Residues in Protein interfaces, a new computational tool to chart hot spots in protein interfaces. Nucleic Acids Res. 2009, 38, e86. [CrossRef]

10. Cho, K.i.; Kim, D.; Lee, D. A feature-based approach to modeling protein-protein interaction hot spots. Nucleic Acids Res. 2009, 37, 2672-2687. [CrossRef]

11. Darnell, S.J.; Page, D.; Mitchell, J.C. An automated decision-tree approach to predicting protein interaction hot spots. Proteins Struct. Funct. Bioinform. 2007, 68, 813-823. [CrossRef]

12. Lise, S.; Archambeau, C.; Pontil, M.; Jones, D.T. Prediction of hot spot residues at protein-protein interfaces by combining machine learning and energy-based methods. BMC Bioinform. 2009, 10, 365. [CrossRef] [PubMed]

13. Huo, S.; Massova, I.; Kollman, P.A. Computational alanine scanning of the 1:1 human growth hormone-receptor complex. J. Comput. Chem. 2002, 23, 15-27. [CrossRef] [PubMed]

14. Rajamani, D.; Thiel, S.; Vajda, S.; Camacho, C.J. Anchor residues in protein-protein interactions. Proc. Natl. Acad. Sci. USA 2004, 101, 11287-11292. [CrossRef] [PubMed]

15. Tuncbag, N.; Gursoy, A.; Keskin, O. Identification of computational hot spots in protein interfaces: Combining solvent accessibility and inter-residue potentials improves the accuracy. Bioinformatics 2009, 25, 1513-1520. [CrossRef]

16. Carrillo-Tripp, M.; Brooks, C.L.; Reddy, V.S. A novel method to map and compare protein-protein interactions in spherical viral capsids. Proteins 2008, 73, 644-655. [CrossRef]

17. Carrillo-Tripp, M.; Montiel-García, D.J.; Brooks, C.L.; Reddy, V.S. CapsidMaps: Protein-protein interaction pattern discovery platform for the structural analysis of virus capsids using Google Maps. J. Struct. Biol. 2015, 190, 47-55. [CrossRef]

18. Carrillo-Tripp, M.; Shepherd, C.M.; Borelli, I.A.; Venkataraman, S.; Lander, G.; Natarajan, P.; Johnson, J.E.; Brooks, C.L.; Reddy, V.S. VIPERdb2: An enhanced and web API enabled relational database for structural virology. Nucleic Acids Res. 2009, 37, D436-D442. [CrossRef]

19. Notredame, C.; Higgins, D.G.; Heringa, J. T-coffee: A novel method for fast and accurate multiple sequence alignment11Edited by J. Thornton. J. Mol. Biol. 2000, 302, 205-217. [CrossRef]

20. Montiel-García, D.J.; Mannige, R.V.; Reddy, V.S.; Carrillo-Tripp, M. Structure based sequence analysis of viral and cellular protein assemblies. J. Struct. Biol. 2016, 196, 299-308. [CrossRef]

21. Katen, S.; Zlotnick, A. The Thermodynamics of Virus Capsid Assembly. In Biothermodynamics, Part A; Methods in Enzymology; Academic Press: Cambridge, MA, USA, 2009; Volume 455, Chapter 14, pp. $395-417$.

22. Reddy, V.S.; Giesing, H.A.; Morton, R.T.; Kumar, A.; Post, C.B.; Brooks, C.L.; Johnson, J.E. Energetics of Quasiequivalence: Computational Analysis of Protein-Protein Interactions in Icosahedral Viruses. Biophys. J. 1998, 74, 546-558. [CrossRef] 
23. Horton, N.; Lewis, N. Calculation of the free energy of association for protein complexes. Protein Sci. 1992, 1, 169-181. [CrossRef] [PubMed]

24. Eisenberg, D.; McLachlan, A.D. Solvation energy in protein folding and binding. Nature 1986, 319, $199-203$. [CrossRef] [PubMed]

25. Zlotnick, A.; Aldrich, R.; Johnson, J.M.; Ceres, P.; Young, M.J. Mechanism of Capsid Assembly for an Icosahedral Plant Virus. Virology 2000, 277, 450-456. [CrossRef] [PubMed]

26. Humphrey, W.; Dalke, A.; Schulten, K. VMD—Visual Molecular Dynamics. J. Mol. Graph. 1996, 14, 33-38. [CrossRef]

27. Ashkenazy, H.; Abadi, S.; Martz, E.; Chay, O.; Mayrose, I.; Pupko, T.; Ben-Tal, N. ConSurf 2016: An improved methodology to estimate and visualize evolutionary conservation in macromolecules. Nucleic Acids Res. 2016, 44, W344-W350. [CrossRef]

28. Lemkul, J.A.; Bevan, D.R. Assessing the Stability of Alzheimer's Amyloid Protofibrils Using Molecular Dynamics. J. Phys. Chem. B 2010, 114, 1652-1660. [CrossRef]

29. Buck, M.; Bouguet-Bonnet, S.; Pastor, R.W.; MacKerell, A.D. Importance of the CMAP Correction to the CHARMM22 Protein Force Field: Dynamics of Hen Lysozyme. Biophys. J. 2006, 90, L36-L38. [CrossRef]

30. Jorgensen, W.L.; Chandrasekhar, J.; Madura, J.D.; Impey, R.W.; Klein, M.L. Comparison of simple potential functions for simulating liquid water. J. Chem. Phys. 1983, 79, 926-935. [CrossRef]

31. Abraham, M.J.; Murtola, T.; Schulz, R.; Páll, S.; Smith, J.C.; Hess, B.; Lindahl, E. GROMACS: High performance molecular simulations through multi-level parallelism from laptops to supercomputers. SoftwareX 2015, 1-2, 19-25. [CrossRef]

32. Torrie, G.; Valleau, J. Nonphysical sampling distributions in Monte Carlo free-energy estimation: Umbrella sampling. J. Comput. Phys. 1977, 23, 187-199. [CrossRef]

33. Kumar, S.; Rosenberg, J.M.; Bouzida, D.; Swendsen, R.H.; Kollman, P.A. THE weighted histogram analysis method for free-energy calculations on biomolecules. I. The method. J. Comput. Chem. 1992, 13, 1011-1021. [CrossRef]

34. Bandyopadhyay, D.; Mehler, E.L. Quantitative expression of protein heterogeneity: Response of amino acid side chains to their local environment. Proteins Struct. Funct. Bioinform. 2008, 72, 646-659. [CrossRef]

35. Marsh, J.A. Buried and Accessible Surface Area Control Intrinsic Protein Flexibility. J. Mol. Biol. 2013, 425, 3250-3263. [CrossRef] [PubMed]

(C) 2019 by the authors. Licensee MDPI, Basel, Switzerland. This article is an open access article distributed under the terms and conditions of the Creative Commons Attribution (CC BY) license (http:/ / creativecommons.org/licenses/by/4.0/). 